Mots. Les langages du politique

$81 \mid 2006$

Suisse, laboratoire politique européen ?

Francis Chateauraynaud, Prospéro. Une technologie littéraire pour les sciences humaines

Johannes Angermuller

\title{
OpenEdition
}

Journals

Édition électronique

URL : https://journals.openedition.org/mots/727

DOI : $10.4000 /$ mots. 727

ISSN : 1960-6001

Éditeur

ENS Éditions

Édition imprimée

Date de publication : 1 juillet 2006

Pagination : 119-121

ISBN : 2-84788-098-4

ISSN : 0243-6450

Référence électronique

Johannes Angermuller, «Francis Chateauraynaud, Prospéro. Une technologie littéraire pour les sciences humaines », Mots. Les langages du politique [En ligne], 81 | 2006, mis en ligne le 01 juillet 2008, consulté le 22 avril 2022. URL : http://journals.openedition.org/mots/727 ; DOI : https://doi.org/10.4000/mots. 727

(c) ENS Éditions 


\section{Comptes rendus de lecture}

\section{Prospéro. Une technologie littéraire pour les sciences humaines}

Francis Chateauraynaud

2003, Paris, CNRS, 406 p.

Les technologies informatiques ont profondément modifié notre rapport aux textes. Grâce à l'ordinateur, de grandes collections de textes sont désormais facilement accessibles. Qu'on utilise des moteurs de recherche généralistes (Google) ou des bases de données spécialisées (LexisNexis, MLA...), l'ordinateur permet de retrouver, stocker et diffuser des textes numérisés en grandes quantités. Si la technologie a considérablement facilité la gestion des données textuelles de masse, on peut en revanche se demander si l'ordinateur peut également contribuer à leur « digestion ».

Aujourd'hui, la gamme de logiciels qui se proposent de donner une réponse à cette question est considérable. Deux philosophies semblent s'opposer à cet égard. La première, proche de la linguistique de corpus et de la lexicométrie, part de la forme graphique nue, ou éventuellement catégorisée, pour découvrir les lois et structures statistiques qui gouvernent leurs distributions, cooccurrences et corrélations (par exemple Lexico 3, Alceste). La seconde, inspirée par la sociologie qualitative telle qu'elle est pratiquée dans les recherches anglosaxonnes et allemandes, part du sens que véhiculent les mots tout en privilégiant leurs interprétations et leurs usages dans des contextes spécifiques. En règle générale, le but de cette dernière est de reconstruire l'univers social et culturel dont sont issus les textes (Qualitative Data Analysis [désormais QDA], WINqda, Atlas.ti). Si les deux approches considèrent le signe comme ayant deux faces - le signifiant et le signifié -, la première met l'accent sur l'examen rigoureux des formes du signifiant tandis que la seconde porte plutôt sur la reconstruction interprétative du signifié.

Développé par l'association Doxa sous la direction de Francis Chateauraynaud, le logiciel Prospéro semble suivre une troisième stratégie, au-delà du formalisme linguistique et du subjectivisme de la QDA. Conçu pour l'analyse des controverses publiques, Prospéro est une collection d'applications qui permet d'analyser des dossiers complexes. À la différence du corpus en lexicométrie, un dossier complexe est un ensemble de données ouvert, hétérogène et dynamique. Rassemblant des textes divers autour d'une thématique, un dossier 
complexe évolue au fur et à mesure qu'on y met de nouveaux textes. Des études se sont ainsi constituées progressivement sur les controverses concernant le nucléaire, l'amiante, le cas Céline. Prospéro exige l'attribution d'un code à chaque mot du corpus, ce qui permet d'établir un vaste réseau conceptuel qui se superpose à l'intégralité des mots du dossier. Ainsi les mots dont la référence est ambigüe sont-ils dotés d'une signification unique ; le syntagme " président de la République » est une unité qui réfère à des personnages différents en 1985 et en 2005. En outre, vu la codification exhaustive, on peut déterminer des présences ainsi que des absences de certains codes dans les textes. Exigeant un travail d'initiation considérable, Prospéro s'inscrit dans le courant de la sociologie interprétative selon laquelle la compréhension est basée sur le travail interprétatif propre aux êtres humains. Mais à la différence de la QDA, c'est ici l'ordinateur qui fait une grande partie de la codification sans pour autant renoncer au travail humain d'interprétation. La codification est fondée sur l'interaction entre l'opérateur et l'ordinateur qui apprend à appliquer les schèmes de codification aux nouveaux textes entrant dans le dossier. Dans la mesure où les textes sont intégrés dans le dossier, les interprétations s'accumulent et la codification exige de moins en moins d'interventions humaines.

Ce livre constitue le premier ouvrage de référence portant sur le projet Prospéro, lequel date d'une dizaine d'années. Il s'efforce donc de présenter le logiciel tout en le situant dans le cadre d'une sociologie générale, voire dans un champ vaste d'investigations contemporaines en sciences humaines. Mais face à l'ambition théorique dont il témoigne, laquelle touche à des champs aussi divers que la nouvelle sociologie, l'informatique, les sciences cognitives, la sémiotique, on peut regretter que la longue première partie ne s'articule pas elle-même avec la description de la méthode Prospéro qui suit. Malgré une discussion sur la méthodologie interprétative de ce logiciel, l'auteur ne le situe pas vraiment dans l'ensemble des logiciels actuels en sciences sociales et en sciences du langage. Quant au fonctionnement technique du logiciel, assez complexe, il n'est traité que dans l'annexe où l'on apprend, en deux pages très rapides, qu'il consiste en quatre modules (Caliban, Ariel, Sycorax, Marlowe) dont le lecteur aurait bien aimé savoir comment ils fonctionnent. Malheureusement, l'auteur ne présente pas sous forme d'un exemple les conditions d'application de Prospéro. Une description systématique d'un cas aurait probablement permis au lecteur de voir quelles sont les problématiques et les questions auxquelles le logiciel peut répondre. Comme il n'y a pas de théorie des objets étudiés, le lecteurn'apprend pas comment, selon l'auteur, définir et circonscrire une controverse publique dans les sociétés contemporaines. On se demande enfin pourquoi ce logiciel est désigné dans le titre comme une «technologie littéraire ». Dans une note de bas de page, l'auteur explique que la partie sur la critique littéraire a dû être enlevée pour " abréger les souffrances du lecteur » (p. 65). 
Malgré ces quelques défauts, il est possible de souligner les points forts de cette méthodologie partiellement automatisée, qui semble réunir des fonctions classiques minimales - de type lexicométrique, de codification - et y adjoindre une partie de type intelligence artificielle :

- automatisation partielle du travail d'interprétation; pour certains discours comme les discours médiatiques, qui se caractérisent par une homogénéité et une structuration, Prospéro semble capable de faire la codification et de repérer la thématique tout seul;

- flexibilité et interactivité du logiciel; quand celui-ci a intégré le codage des textes, il est facile d'élargir un dossier. Effectivement, le point fort du logiciel est de confronter systématiquement l'opérateur avec les éléments que le logiciel n'arrive pas à comprendre. L'opérateur peut ainsi toujours revenir au travail d'interprétation précédent;

- caractère ouvert des dossiers; avec Prospéro, il est possible de traiter des dossiers qui n'ont pas de limites naturelles, ce qui est le cas pour à peu près tous les phénomènes sociaux. Le logiciel est particulièrement apte à rendre compte des évolutions actuelles dans les discours médiatiques;

- la dimension thématique de l'analyse s'appuie sur de nombreuses fonctions qui permettent de décrire un dossier au niveau de la forme graphique ainsi qu'aux niveaux thématique et sémantique. C'est ce dernier point qui devrait intéresser surtout les chercheurs en sciences sociales: ils disposent ainsi d'un logiciel qui leur permet de se rendre compte du contenu des textes.

$\mathrm{Si}$ ce livre ne réalise pas toutes ses ambitions, il introduit néanmoins à un logiciel qui vise à réconcilier les approches interprétatives avec l'analyse automatique assistée par ordinateur et qui présente des solutions à plusieurs problèmes techniques pour l'analyse thématique des textes, en intégrant des fonctions de l'intelligence artificielle dans le dépouillement des textes et en créant un ensemble d'applications dont il faut souligner la variété des fins possibles. Il manque néanmoins encore un cadre conceptuel qui lierait les problèmes et les questions des sciences sociales avec le potentiel d'analyse du logiciel. II faudra attendre la parution d'autres publications pour donner une idée de ce que peut apporter Prospéro aux sciences humaines et sociales.

Johannes Angermüller

Université Paris 12, Céditec

johannes.angermueller@gse-w.uni-magdeburg.de 